\title{
PlioVAR marine synthesis update meeting
}

\author{
Erin L. McClymont ${ }^{1}$, A.M. Haywood ${ }^{2}$, P. Dekens ${ }^{3}$, A. Rosell-Melé ${ }^{4}$ and L. Dupont ${ }^{5}$
}

Utrecht, The Netherlands, 2 September 2016

Following the International Conference on Paleoceanography in Utrecht, 40 members of the PlioVAR working group met to receive and discuss progress reports on synthesis work of existing and emerging multi-proxy marine data during the late Pliocene. Participants working with marine proxies and with varied regional interests were present, as well as those with previous experience in large paleoclimate syntheses. Six presentations were given, with the first outlining the aims and objectives of PlioVAR. The other five reported on the developing syntheses from the North Atlantic, South Atlantic, Pacific, Indian and Southern Oceans. An open discussion then considered the implications of the work so far, alongside future strategies to support continued synthesis. The questions and priorities identified during the meeting include:

\section{Regional differences emerging in glacial-interglacial variability}

A target of PlioVAR is to assess the magnitude and rate of climate changes associated with the "M2-KM3" event, marked by ice-sheet expansion and then retreat around 3.3-3.2 Ma BP (Fig. 1). This climate transition was identified in many of the existing records, but its amplitude varied depending on local circulation (e.g. upwelling, proximity to fronts), water mass and the proxy used. The importance of setting the late Pliocene variability in the context of Pliocene-Pleistocene climate evolution was noted, and the potential to examine shorter timescale variability was outlined. Variations in dust supply and glacial inputs to marine sediments were also noted as potentially valuable indicators of atmospheric circulation, environmental change onshore and ice-sheet behavior.

\section{Reconciling environmental signals from multi-proxy analyses}

A key aim of PlioVAR is to draw together multi-proxy perspectives on climate and environmental variability. Discussion about the signals recorded by different temperature proxy records emphasized the importance of detailing proxy measurements and interpretations in both the original publications and in the PlioVAR synthesis. These issues are not unique to PlioVAR but the working group is mindful of the implications, especially for planned data-model intercomparison.

\section{New insights expected from} recent and planned ocean drilling Several recent IODP expeditions have secured new archives which have great

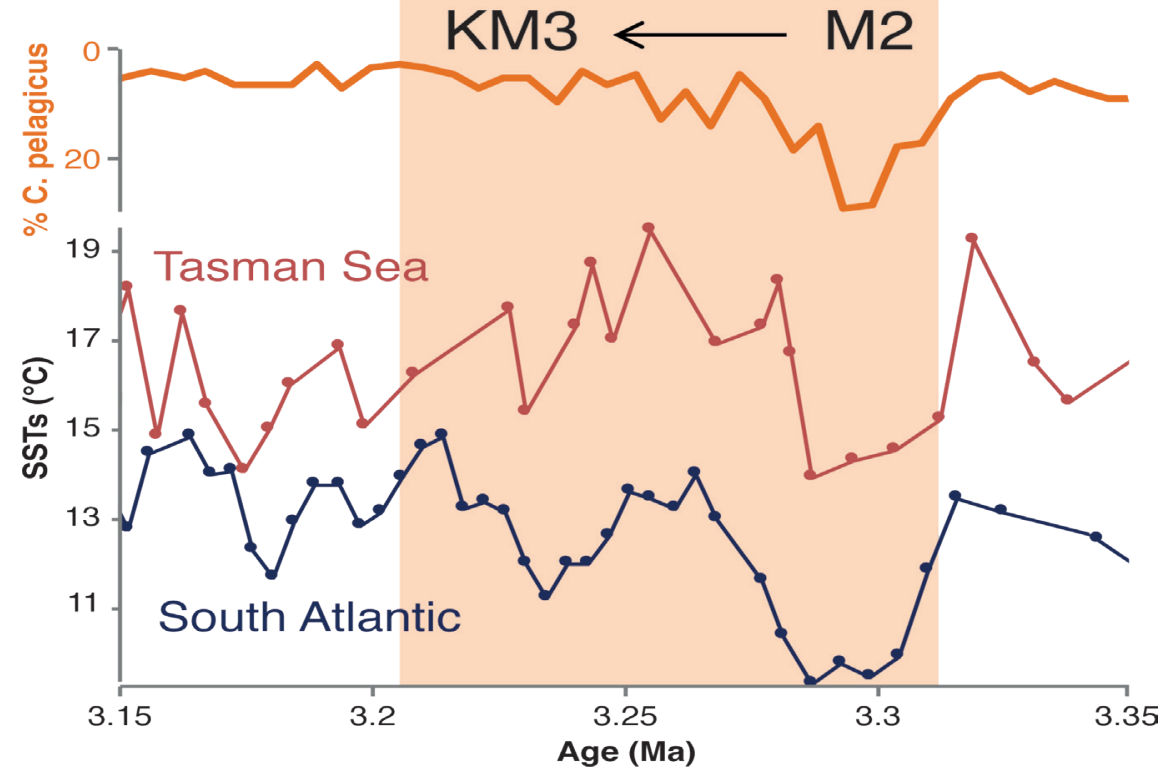

Figure 1: An example of data synthesis and comparison for the $\mathrm{M} 2-\mathrm{KM} 3$ transition from three southern hemisphere records: abundance of coccoliths from Coccolithus pelagicus at ODP Site 1172 (east of Tasmania; Ballegeer et al. 2012), and reconstructed sea-surface temperatures (SSTs) from DSDP Site 593 (Tasman Sea; McClymont et al. 2016) and ODP Site 1090 (Southern Atlantic; Martinez-Garcia et al. 2010).

potential to contribute to the PlioVAR initiative, in areas where data is currently limited or absent (e.g. Exp 353 Indian Monsoon, Exp 354 Bengal Fan, Exp 355 Arabian Sea Monsoon, Exp 356 Indonesian Throughflow, Exp 361 Southern African Climates). Several members of the science parties for these expeditions were present, and future plans and preliminary data were noted.

\section{Management and integration of published and emerging data}

Several presentations outlined the challenges of identifying and downloading existing data for incorporation into the synthesis, particularly due to missing information about stratigraphy, proxy calibrations and interpretations, as well as data not being available post-publication. Recommendations for data formatting and upload were discussed, with a need for metadata (e.g. which calibration was applied, IODP sample identifiers plus depth scales, original not just calibrated data provided). Ongoing efforts to create a protocol for assessing the quality of the age control were noted. Several possible means of creating an interface to view and download available data were presented, and their potential will be explored further

Several conference sessions with a PlioVAR focus are scheduled, for example at the AGU Fall Meeting (2016) and PAGES Open Science Meeting (2017). Anyone interested in contributing to PlioVAR is encouraged to participate. The next PlioVAR meeting (19-21 April 2017, in Durham, UK; www.pastglobalchanges.org/calendar/127-pages/1658-pliovar-wshop-apr-17) will take place shortly before the PAGES OSM to focus on advancing the marine synthesis efforts outlined here. More information will be transmitted through the PAGES website and via the working group mailing list (www.pastglobalchanges.org/ini/wg/pliovar/).

We thank PAGES for the funding support which made this meeting possible.

\section{AFFILIATIONS}

${ }^{1}$ School of Geography, Durham University, UK ${ }^{2}$ School of Earth \& Environment, University of Leeds, UK ${ }^{3}$ Department of Geosciences, San Francisco State University, USA

${ }^{4}$ ICREA and Universitat Autònoma de Barcelona, Spain ${ }^{5}$ MARUM - Center for Marine Environmental Sciences, University of Bremen, Germany

\section{CONTACT}

Erin McClymont: erin.mcclymont@durham.ac.uk REFERENCES

Ballegeer A-M et al. (2012) Palaeogeog Palaeoclimatol Palaeoecol 313-314: 215-224

Martinez-Garcia A et al. (2010) Science 328: 1550-1553

McClymont EL et al. (2016) Paleoceanography 31: 895-913 